\title{
Intérêt thérapeutique de la superoxyde dismutase liposomiale (Lipsod) dans les complications fibreuses induites par la radiothérapie ( $R T$ )
}

\author{
S. DELANIAN, F. BAILLET, M. HOUSSET \\ Hôpital Necker et Hôpital Salpétrière Paris APHP, \\ 149, rue de Sèvres, 75743 Paris Cedex 15
}

Les séquelles radioinduites sont classiquement irréversibles. Après échec des corticoïdes, un traitement par Lipsod a été administré sur 3 semaines chez 69 patients en 6 injections IM de 5, 2 ou $1 \mathrm{mg}$ chacune, dans 3 circonstances:

(a) 45 radiofibroses mesurables de $8,5 \mathrm{~cm}(3-24)$ dans le plus grand axe ont été traitées 5 ans en moyenne après $\mathrm{RT}$; un ramollissement clinique majeur a été observé dans $80 \%$ parallèlement à une amélioration fonctionnelle, puis une régression fibreuse $>1 / 3$ dans $42 \%$; aucun effet n'a été observé sur l'atrophie cutanée ou un déficit neurologique associé ;

(b) 20 patients avec asialie clinique et scintigraphique (2,5 ans/RT ORL) ont présenté, après 1 ou 2 cures, une amélioration franche (35\%) ou une quasi normalisation salivaire (45\%) ;

(c) 4 patients avec grêle radique (dans six mois/RT) ont présenté, après 2 cures à 2 mois d'intervalie, une réponse importante et 2 améliorations franches maintenues à 3 ans, tandis que la réponse modeste a rechuté.

Ainsi la Lipsod permet de moduler les effets adverses récents et tardifs de la RT. Sa tolérance est bonne : $10 \%$ de fièvre à $38^{\circ} 5 \mathrm{C}$ transitoire. Son efficacité est rapide (dès la fin des injections avec maximum à 2 mois), stable dans le temps (recul de 2-7 ans), reproductible (après $2^{e}$ cure), sans effet dose (dans les limites étudiées). 